\title{
Assessing the severity of functional impairment of psychiatric disorders: equipercentile linking the mini-ICF-APP and CGI
}

\author{
Stephan T. Egger ${ }^{1,2^{*}}$ (D) Godehard Weniger ${ }^{1}$, Mario Müller ${ }^{1}$, Julio Bobes ${ }^{2}$, Erich Seifritz ${ }^{1}$ and Stefan Vetter ${ }^{1}$
}

\begin{abstract}
Background: The assessment of functioning and impairment due to psychiatric illness has been acknowledged to be crucial for research and practice. This led to the development of the mini-ICF-APP, which provides a reliable and time-efficient measure of functioning and impairment. Although its use is increasing, it remains unclear how it reflects severity and how change might be interpreted from a clinical perspective.

Methods: In a clinical sample of 3067 individuals hospitalized for mental health treatment, we used an equipercentile approach to link the mini-ICF-APP with the Clinical Global Impression scale (CGI) at admission and discharge. We linked the mini-ICF-APP sum score to the CGI-S scale and the mini-ICF-APP proportional change between admission and discharge to the CGI-I scale.

Results: The mini-ICF-APP and CGI scales showed a Spearman correlation of $0.50(p<.000)$. CGI-S: "borderline-ill" corresponded to a mini-ICF-APP score 1-2; "mildly-ill" to 3-7; "moderately-ill" to 8-15; "markedly-ill" to 16-24; "severely-ill" to 25-37; and "extremely-ill" to a score $\geq 38$. The Spearman correlation between the percentage change of mini-ICF-APP sum score and the CGI-I was 0.32 ( $p>$.000); "no-change" in the CGI-I corresponded to an increase or decrease of 2\%; "minimally-improved" to a mini-ICF-APP reduction of 3-30\%; "much-improved" to a reduction of 31-63\%; "very-much-improved" to a reduction of $\geq 64 \%$ "minimally-worse" to an increase of 3-34\% "much-worse" to an increase of 35-67\%; and finally "very-much-worse" with an increase of $\geq 68 \%$.
\end{abstract}

Conclusions: Our findings improve understanding of the clinical meaning of the mini-ICF-APP sum score and percentage change in patients hospitalized for treatment.

Keywords: Psychiatric disorders, Functionality, Severity, Mini-ICF-APP, CGl, Equipercentile

\section{Background}

Mental and substance use disorders contribute substantially to the global burden of disease, being, directly and indirectly, responsible for a significant amount of mortality and morbidity [1]; to a large extent through delayed access to treatment and services [2]. Furthermore, the impact of mental health on disability and incapacity is on the rise [3-5]. Mental disorders are a huge challenge for public health,

\footnotetext{
*Correspondence: stephan.egger@puk.zh.ch

${ }^{1}$ Centre for Integrative Psychiatry, Department of Psychiatry, Psychotherapy and Psychosomatics, Psychiatric University Hospital of Zurich, University of Zurich, Lenggstrasse 31, 8032 Zurich, Switzerland

${ }^{2}$ Department of Psychiatry, Faculty of Medicine, University of Oviedo, CIBERSAM, Oviedo, Spain
}

compounded by the challenges of psychiatric diagnosis itself [1]. In contrast to most other fields of medicine, psychiatric diagnosis remains primarily restricted to subjective symptoms and observable signs [6]; furthermore, there is a poor correspondence between impairment and symptom load [7-10]. This has led to ongoing controversy about the usefulness of psychiatric diagnoses, as well as their reliability and validity $[1,10,11]$.

Accordingly, the WHO (World Health Organisation) introduced the ICF (International Classification of Functionality), designed to assess the particular mix of strengths, weaknesses, and other circumstances which may affect the course of a patient's illness and treatment

(c) The Author(s). 2019 Open Access This article is distributed under the terms of the Creative Commons Attribution 4.0 International License (http://creativecommons.org/licenses/by/4.0/), which permits unrestricted use, distribution, and reproduction in any medium, provided you give appropriate credit to the original author(s) and the source, provide a link to the Creative Commons license, and indicate if changes were made. The Creative Commons Public Domain Dedication waiver (http://creativecommons.org/publicdomain/zero/1.0/) applies to the data made available in this article, unless otherwise stated. 
response, independent of actual symptom levels [12]. However, the ICF is far too complex and timeconsuming to be implemented in everyday clinical practice $[7,13]$. The mini-ICF-APP was developed in accordance to the WHO-ICF parameters; it is a 13-item short observer-rating instrument to assess functioning and impairment in persons suffering from mental health problems, irrespective of diagnosis [13].

The original version, as well as the first studies involving the mini-ICF-APP were validated on patients with a nonpsychotic mental disorder hospitalized for rehabilitation treatment [13-15]. The subsequent studies of the English and Italian version of the scale covered a broader diagnostic spectrum, including patients with a psychotic disorder [16-18]. Recent studies focused on patients with a chronic mental disorder [19] as well as with posttraumatic stress disorder [20]. Since its introduction, the mini-ICF-APP has been progressively implemented by health care providers, insurance companies and pension funds to assess disability and work impairment [16-18, 21, 22].

The evaluation of functioning and disability has vast implications for those affected since it may impact access to services and financial support (e.g., disability pension). Despite a number of validation studies [13, 16-18], there is limited support for the clinical usefulness and interpretation of the mini-ICF-APP. Although higher scores imply greater severity, its clinical relevance is not fully understood. The Clinical Global Impression Scales (CGI), in contrast, describes the overall clinical impression regarding severity or improvement [23]. Therefore, the CGI has been previously used to establish the usefulness of other clinical measures [24-26]. This study aims to provide clinically meaningful and valid scores for the mini ICF which correspond statistically to CGI levels in a sample of patients hospitalized for treatment.

\section{Methods}

\section{Sample and procedure}

The Centre for Integrative Psychiatry, as part of the Psychiatric University Hospital of Zurich, offers a specialized psychiatric and psychotherapeutic treatment program for patients for whom outpatient treatment alone is insufficient to achieve improvement. We used data from a full five-year cohort of consecutive patients hospitalized for treatment $(n=3295)$. Seven percent $(6.91 \%=228)$ of the sample excluded due to one or more missing ICF items. Missing data did not differ from the remaining sample regarding sex, age, education, marital status or primary diagnosis (data not shown). The final sample used for subsequent analyses comprised 3067 patients. The data collected is part of the routine clinical care; the competent ethics committee approved the use of this data for further analysis and publication [KEK-ZH BASEC-Nr. 2017-01766].

\section{Raters and training}

Diagnoses were made by a senior psychiatrist according to the ICD-10 diagnostic criteria [27]. Raters were clinicians; psychiatrists, psychiatry residents or clinical psychologists. All raters were trained regularly trained and supervised on the use of the measures employed in this study. All study measures were rated by the same rater, considering the past seven days. Information was obtained through clinical interviews and direct behavioral observation; or provided by nursing staff, social workers and significant others who were directly involved in the treatment process.

\section{Diagnostic groups}

We defined nine diagnostic groups in accordance with ICD-10 classifications [27]: NCD: Neurocognitive Disorders (ICD-10: F0); AUD: Alcohol Use Disorders (ICD-10: F10); SUD: Substance Use Disorders (ICD-10: F11-F19); SPD: Schizophrenia and other psychotic disorders (ICD-10: F2); BPD: Bipolar Disorders (ICD-10: F31); MDD: Major Depressive Disorders (ICD-10: F32 and F33); AXD: Anxiety Disorders (ICD-10: F4X); PD: Personality Disorders (ICD10: F6); and NDD: Neurodevelopmental Disorders (ICD-10: F7; F8 and F9).

\section{Measures}

The Clinical Global Impression (CGI) scale is a brief, easy to use and pragmatic tool for the assessment of psychiatric illness severity and changes over time, which is intuitively understood and widely used in clinical practice [23, 28-32]. The CGI consists of three subscales: 1. Severity of Illness (CGI-S), 2. Global Improvement (CGI-I), and 3. Efficacy Index (CGI-E); which will not be used [28]. CGI-S and CGI-I have a seven-point Likert scale response format ranging from 1 representing the "healthy subject" to 7 the "extremely ill subject." The CGI-I ranges from 1 "significant improvement" to 7 "most severe deterioration," whereby a score of 4 indicates no change [28]. Ratings of CGI-S and CGI-E refer to the past week, ratings of CGI-I to the time elapsed since the first CGI-S assessment. Consequently, CGI-I assessed at the time of discharge from treatment.

\section{Mini ICF-APP}

The International Classification of Functioning, Disability, and Health (ICF) [33] was introduced by the WHO to supplement the primarily symptom-related description of diseases, such as the ICD-10 [27]. The ICF describes and classifies disorders according to the level of functioning, activity/capacity and context, which are directly or indirectly related to restrictions in social participation. The use of the ICF in clinical practice is restricted due to its complexity and time-consuming nature. The mini-ICF-APP [13], was developed as a short 
observer-rated scale to assess the level of functioning. The mini-ICF-APP consists of thirteen domains of functioning: (1) adherence to regulations and routines, (2) planning and structuring of tasks, (3) flexibility, (4) competency/efficacy, (5) endurance, (6) assertiveness, (7) contact with others, (8) group integration, (9) family and intimate relationships, (10) leisure activities, (11) selfcare, (12) mobility and (13) competence to judge and decide. Each item is rated on a five-point Likert scale from 0 (no disability) to 4 (total disability); with anchor definitions for each item provided in the manual. The qualifying of capacities has to be done in reference to a specified context (e.g. work place, work in general, household, etc.). The scale ranges from 0 to 52 points. The mini-ICF-APP is validated and has good psychometric properties $[13,14,16,17]$.

\section{Statistical analysis}

The analysis included only subjects with complete CGI and mini ICF data available at baseline. Simple descriptive statistics were used to represent the demographic and clinical characteristics of the sample. We used a multivariate regression to determine correlation between demographic and clinical variables (gender, age, education and main diagnosis) and the variables used for equipercentile analysis. For the qualitative variables we used the kernel smoothing method for mixed data types. Spearman's rank coefficient used for pairwise correlations. Equipercentile analysis was used to compare the mini-ICF-APP scale with the CGI scales. In equipercentile linking corresponding cut-points on two different scales are determined, it does this by identifying scores on both measures that have the same percentile rank $[34,35]$. In a first step the distribution of both study measures was generated; in a second step distributions were log-linear smoothed, and the corresponding percentiles of both tests matched in the following manner: The CGI-S was linked to the sum score of the mini-ICFAPP; while the CGI-I to the percentage change of the mini-ICF-APP score, between admission and discharge. Anchor values were defined by a CGI-I score of 4 ("no change") and a mini-ICF-APP percentage change of zero ("0"). Statistical analyses conducted by the statistical software R (v3.5.1), for multivariate regression we used the package "np" (v0.60-9); for equipercentile calculation we used the package "equate" (v2.0.7) [34].

\section{Results}

The study population had an age between 16 and 77 years ( $43.4 \pm 11.9$ years); $66.16 \%$ were male. The majority of the sample was single (53.14\%), had started apprenticeship or college/university education (67.53\%). Patient admission was primarily voluntary (94.95\%) with a mean length of stay of $40.57 \pm 32.46$ days. Almost all individuals were routinely discharged $(73.62 \%)$. The most common diagnosis was AUD (53.60\%), followed by SPD (15.29\%). A summary of the demographic characteristics of the complete sample is in Table 1. At admission the mini ICF sum score was $19.20 \pm 9.89$; the CGI- $\mathrm{S}$ was $5.31 \pm 0.89$. At discharge the mini-ICF-APP score was $16.04 \pm 9.55$; the CGI-S score was $4.73 \pm 1.16$; the CGI-I score $2.75 \pm 0.96$.

\section{Multivariate regression}

The regression model found a statistic significant correlation between the mini-ICF-APP sum score and the CGI$\mathrm{S}$ score $(p<0.001)$; as well as for the mini-ICF-APP percentage of change and the CGI-I score $(p<0.001)$. Both correlations were independent of gender; age; diagnosis, education or civil status; with $p$ values over the significance threshold of 0.05 for all variable levels. In addition the correlation between percentage of change and improvement was independent from the initial severity.

Table 1 Subject Demographics and main diagnosis

\begin{tabular}{|c|c|}
\hline \multicolumn{2}{|l|}{ Variable } \\
\hline Age in years mean, (SD) & $43.4(11.9)$ \\
\hline Female $n,(\%)$ & $1038(33.84)$ \\
\hline \multicolumn{2}{|l|}{ Civil Status n, (\%) } \\
\hline Single & $1691(55.14)$ \\
\hline Married & $512(16.70)$ \\
\hline Separated & $128(4.17)$ \\
\hline Divorced & $677(22.07)$ \\
\hline Widowed & $54(1.76)$ \\
\hline Unknown & $5(0.16)$ \\
\hline \multicolumn{2}{|l|}{ Education $n$, (\%) } \\
\hline Primary School & $777(25.33)$ \\
\hline Secondary School & $98(3.19)$ \\
\hline Apprenticeship & $1714(55.89)$ \\
\hline College/University & $357(11.64)$ \\
\hline Unknown & $121(3.95)$ \\
\hline \multicolumn{2}{|l|}{ Main Diagnosis n, (\%) } \\
\hline NCD & $45(1.47)$ \\
\hline AUD & $1644(53.60)$ \\
\hline SUD & $135(4.40)$ \\
\hline SPD & $469(15.29)$ \\
\hline BPD & $80(2.61)$ \\
\hline MDD & $272(8.87)$ \\
\hline$A X D$ & $110(3.59)$ \\
\hline PD & $276(9.00)$ \\
\hline NDD & $36(1.17)$ \\
\hline
\end{tabular}

Legend: NCD Neurocognitive Disorders, AUD Alcohol Use Disorders, SUD Substance Use Disorders (except AUD), SPD Schizophrenia and other psychotic disorders, BPD Bipolar Disorder, MDD Major Depressive Disorder, AXD Anxiety Disorder, $P D$ Personality Disorders, and NDD Neurodevelopmental Disorders 


\section{Linking CGI-S and the mini-ICF-APP sum score}

We found a Spearman correlation coefficient between the CGI-S and the mini-ICF-APP sum score at admission of $0.50(p<.001)$ for the total sample. Diagnostic groups were found to have significant correlations coefficients (between 0.44 and 0.67); except for NDD (0.16, n.s.). After excluding NDD the total sample correlation was 0.51 $(p<.001)$. At discharge, the correlation between the CGI$\mathrm{S}$ and mini-ICF-APP sum score was $0.54(\mathrm{p}<.001)$. The CGI-S score of 2 ("borderline ill") corresponded to a miniICF-APP score between 1 and 2; CGI-S scores of 3 ("mildly ill") to a mini-ICF-APP score between 3 and 7; CGI- S scores of 4 ("moderately ill") to mini-ICF-APP scores between 8 and 15; CGI-S scores of 5 ("markedly ill") to mini-ICF-APP scores between 16 and 24; CGI-S score of 6 ("severely ill") to mini-ICF-APP scores between 25 and 37; finally CGI-S score of 7 ("extremely ill") to a mini-ICF-APP score of 38 or more. Cut-off values are summarized in Table 2 and represented in Fig. 1.

\section{Linking CGI-I and the mini-ICF-APP percentage change}

The Spearman correlation coefficient between the CGI-I and the mini-ICF-APP sum score difference was $0.30(p<.001)$; and between the CGI-I and the percentage change of miniICF-APP sum score, $0.32(\mathrm{p}<.001)$. The CGI-I score of 4 ("no change") was linked to a percentage increase or decrease of 2 percentage points in the mini-ICF-APP; the CGI-I score of 3 ("minimally improved") to a percentage reduction between 3 and 30 points; the CGI-I score of 2 ("much improved") to a percentage reduction between 31 and 63 points; the CGI-I score of 1 ("very much improved") to a percentage reduction of 64 or more; a CGI-I score of 5 ("minimally worse") to a percentage increase between 3 and 34 points; a CGI-I score of 6 ("much worse") with a percentage increase between 35 and 67; and finally a CGI-I score of 7 ("very much worse") with a percentage increase of 68 or more. Cut-off values are summarized in Tables 2 and 3.

\section{Discussion}

This approach of equipercentile linking allows the definition of severity cut-off values for the mini-ICF-APP

Table 2 Cut- off values for the mini-ICF-APP sum score according to CGI-S

\begin{tabular}{ll}
\hline CGI- S & mini-ICF-APP sum score \\
\hline Normal & $0-1$ \\
Borderline III & $1-2$ \\
Mildly III & $3-7$ \\
Moderately III & $8-15$ \\
Markedly III & $16-24$ \\
Severely III & $25-37$ \\
Extremely III & $>38$ \\
\hline
\end{tabular}

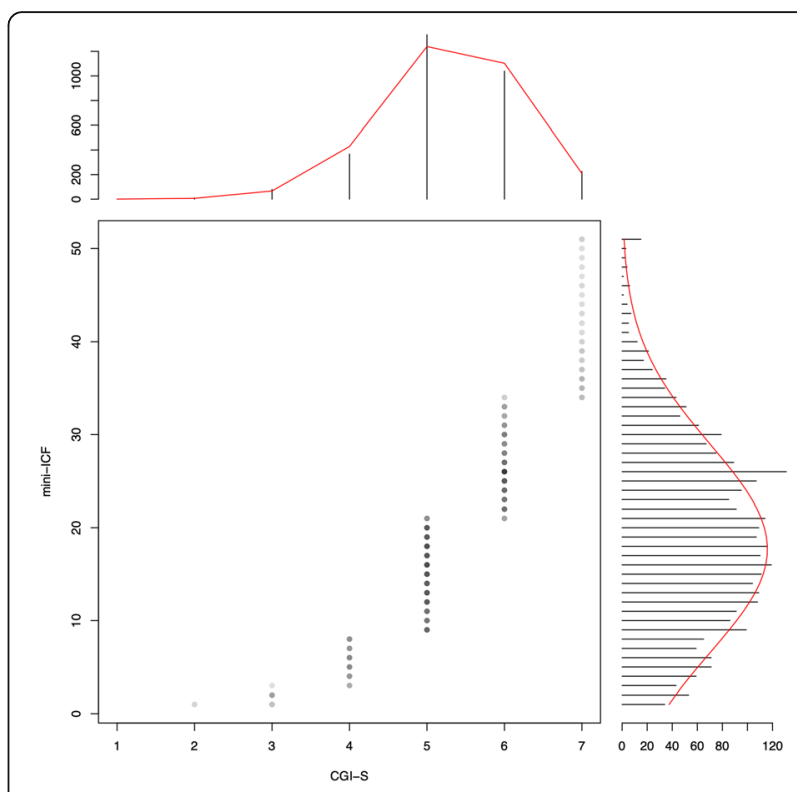

Fig. 1 Equipercentile linking of the mini-ICF-APP sum score and the CGI-S. CGI-S values corresponding: 1: "Normal"; 2. "Borderline III"; 3. "Mildly III"; 4. "Moderately III" 5. "Markedly ill"; 6. "Severely ill"; and 7. "Extremely ill"

according to clinical judgment. Taken together this provides a better understanding and interpretation of the mini-ICF-APP in day-to-day clinical practice. This is of singular importance since the mini-ICF-APP is increasingly being used by health insurance companies and pension funds to determine disability or incapacity benefits caused by a psychiatric condition [21]. Our large clinical sample included diagnoses underrepresented in prior studies with the inclusion of: alcohol and substance use disorders; personality disorders; neurocognitive and neurodevelopmental disorders.

The results of the multivariate analysis did not show a statistical correlation between the mini-ICF-APP and CGI scales and demographic and clinical variables (age, gender, education and main diagnosis). Between the mini-ICF and CGI we were able to show a strong statistical correlation. The correlations found show that illness severity at large

Table 3 Cut- off values for the percentage change according to the CGI-I

\begin{tabular}{ll}
\hline CGI- I & mini-ICF-APP percentage \\
\hline Very much improved & $<63$ \\
Much Improved & $(-) 30-62$ \\
Minimally Improved & $(-) 2-29$ \\
No Change & 0 \\
Minimally Worse & $(+) 2-33$ \\
Much Worse & $(+) 34-66$ \\
Very much worse & $>67$ \\
\hline
\end{tabular}


is associated with capacity limitations in general. The pairwise comparison by of the scales according to the main diagnosis, produce similar associations between both scales for practically all diagnostic groups. In our analysis age, gender, education and main diagnosis seemingly have no effects on the anchor points between the mini-ICFAPP and CGI scales. Furthermore, change and improvement were independent of the baseline severity. Therefore, we consider that the mini-ICF-APP can be viewed as a universally applicable scale for a diagnosis-independent judgment of functionality; broadening the applicability of the scale.

The mini-ICF-APP severity cut-off points we propose for the mini-ICF-APP are roughly comparable with the values reported in previous studies [16-18, 21, 36]. A direct contrast is however not possible, since the approach to severity assessment differs; with either different gradings of severity [36]; gross allocation of severity grades to mini-ICF-APP values $[16,18]$; or the use of psychosocial attributes of severity [17, 21]. Our findings regarding the percentage change are in line with previous findings using the mini-ICF-APP to assess the effectiveness of treatment [14]. Furthermore, our results also concord with the general (although controversially discussed) parameters used to judge improvement [37, 38], were a reduction of $20-30 \%$ is considered an indication of response to treatment while a reduction greater than $50 \%$ is seen as a significant improvement $[39,40]$. The difficulties comparing severity rankings and improvement are not surprising since most available studies dealt with validation issues [13, 16-18].

The severity of a disorder has waste clinical implications, it influences decisions regarding the type and intensity of treatment, whether to continue or stop treatment, but also affect the assess to assistance or disability benefits [41]. The assessment of severity in psychiatric disorders is, however, an issue of ongoing debate, with no resolution insight $[41,42]$. The CGI is considered a pragmatic and intuitive scale [23]; that is commonly used to approach severity and change [41, 43-45]. The CGI is considered useful and necessary in routine clinical practice as well as research settings [32]; as well as previously used for the determination of severity cut-off values [24, 26, 46, 47].

Equipercentile linking, allows for a nominal translation from one scale to another, identifying those scores on both scales which have the same percentile ranks [35]. It's is used in testing programs that involve multiple test forms, like in the educational system [34, 35]. Equipercentyle analysis does not require a specific distribution type and allows for possible measurement errors on both scales compared [35], for this reason, it is considered the preferred linking method amongst other available methods [47, 48]. With an increasingly large number of published studies using this method comparing scales in the fields of psychiatry and neurology [49-52]; with the CGI widely used as a determinant of severity ranking [24-26, 46, 47, 53, 54].

The main strength of our study is the large sample population, without inclusion or exclusion criteria, with a broad diagnostic range, including diagnoses underrepresented in previous studies. The similar correlation coefficients and linking values for the different diagnostic categories confirms the use of the mini-ICF-APP independent of diagnosis $[13,16,17]$. The inclusion of one study site determines some peculiarities in the sample studied. In our case, the large number of patients with AUD is, due to a detoxification treatment program, with a high admission capacity. The over-representation of male patients might be a potential limitation; however, neither the regression model nor the post hoc analysis revealed an effect of gender on the results. One limitation of our study is the recruitment of the sample from patients hospitalized for treatment, which limits the interpretation of the data to these patients requiring treatment. In this context also the influence of the different health systems on treatment seeking behaviour and utilization of mental health services should considered [55].

Our results should be interpreted with caution; since the mini-ICF-APP sum score does not reflect a global incapacity index. Patients with limitations in certain dimensions still could experience impairment; due to the repercussion in other domains. Furthermore, it also should consider how the individual human beings deal with incapacity and seek for solutions on their own. We consider that the CGI scales take account all of these phenomena; with the resulting rather exponential correlation between both scales.

\section{Conclusions}

The results demonstrate in general that illness severity is associated with capacity limitations and that functional change corresponds to a clinical impression of improvement. Our findings contribute to a deeper understanding and clinical interpretation of the mini-ICF-APP.

\section{Abbreviations \\ AUD: Alcohol Use Disorders; AXD: Anxiety Disorders; BPD: Bipolar Disorders; CGI: Clinical Global Impression Scales; ICD: International Classification of Disease; ICF: International Classification of Functionality; MDD: Major Depressive Disorders; NCD: Neurocognitive Disorders; NDD: Neurodevelopmental Disorders; PD: Personality Disorders; SPD: Schizophrenia and other psychotic disorders; SUD: Substance Use Disorders; WHO: World Health Organisation}

\section{Acknowledgments \\ Not applicable.}

\section{Authors' contributions}

STE data analysis and writing of the manuscript; GW project conception and writing of the manuscript; MM data management and data analysis; JB analysis design, interpretation of data and substantial revision of the manuscript; ES interpretation of data and substantial revision of the 
manuscript; SV project design and substantial revision of the manuscript. Al authors read and approved the final manuscript.

\section{Funding}

None.

\section{Availability of data and materials}

The protocol presented and approved by the ethics committee does not foresee to share the data. On reasonable request, the corresponding author will submit an amendment to the ethics committee asking for approval of data sharing in that particular case.

\section{Ethics approval and consent to participate}

Data was routinely collected as part of a treatment quality program. The further use of the clinical data was authorized by the competent ethics committee of Zurich, Switzerland [KEK-ZH BASEC-Nr. 2017-01766].

\section{Consent for publication}

Not applicable.

\section{Competing interests}

The authors declare that they have no competing interests.

Received: 10 January 2019 Accepted: 18 October 2019

Published online: 19 November 2019

\section{References}

1. Lynskey MT, Strang J. The global burden of drug use and mental disorders. Lancet. 2013;382(9904):1540-2.

2. Wang PS, Angermeyer M, Borges G, Bruffaerts R, Chiu WT, De Girolamo G, et al. Delay and failure in treatment seeking after first onset of menta disorders in the World Health Organization's world mental health survey initiative. World Psychiatry. 2007:6(3):177.

3. Whiteford HA, Degenhardt L, Rehm J, Baxter AJ, Ferrari AJ, Erskine HE, et al. Global burden of disease attributable to mental and substance use disorders: findings from the global burden of disease study 2010. Lancet. 2013:382(9904):1575-86.

4. Vos T, Allen C, Arora M, Barber RM, Bhutta ZA, Brown A, et al. Global, regional, and national incidence, prevalence, and years lived with disability for 310 diseases and injuries, 1990-2015: a systematic analysis for the global burden of disease study 2015. Lancet. 2016;388(10053):1545-602.

5. Kassebaum NJ, Arora M, Barber RM, Bhutta ZA, Brown J, Carter A, et al. Global, regional, and national disability-adjusted life-years (DALYS) for 315 diseases and injuries and healthy life expectancy (HALE), 1990-2015: a systematic analysis for the global burden of disease study 2015. Lancet. 2016;388(10053):1603-58.

6. Insel TR, Cuthbert BN. Brain disorders? Precisely Science. 2015;348(6234): 499-500.

7. Linden M. Disease and disability. The ICF model. Nervenarzt. 2015;86(1):29-35

8. Kendler KS. Setting boundaries for psychiatric disorders: editorial. Am J Psychiatr. 1999;156(12):1845.

9. Angold A, Costello EJ, Farmer EMZ, Burns BJ, Erkanli A. Impaired but undiagnosed. J Am Acad Child Adolesc Psychiatry. 1999;38(2):129-37.

10. Timimi S. No more psychiatric labels: why formal psychiatric diagnostic systems should be abolished. Int J Clin Health Psychol. 2014;14(3):208-15.

11. Frances AJ, Nardo JM. ICD-11 should not repeat the mistakes made by DSM-5. Br J Psychiatry. 2013;203(1):1-2.

12. Cuthbert $B$, Insel $T$. The data of diagnosis: new approaches to psychiatric classification. Psychiatry. 2010:73(4):311-4.

13. Linden M, Baron S. The "mini-ICF-rating for mental disorders (mini-ICF-P)". A short instrument for the assessment of disabilities in mental disorders. Rehabilitation. 2005;44(3):144-51.

14. Baron S, Linden M. Analyzing the effectiveness of inpatient psychosomatic rehabilitation using the mini-ICF-APP. Rehabilitation. 2009:48(3):145-53.

15. Linden M, Baron S, Muschalla B. Relationship between work-related attitudes, performance and capacities according to the ICF in patients with mental disorders. Psychopathology. 2010;43(4):262-7.

16. Balestrieri M, Isola M, Bonn R, Tam T, Vio A, Linden M, et al. Validation of the Italian version of mini-ICF-APP, a short instrument for rating activity and participation restrictions in psychiatric disorders. Epidemiology and psychiatric sciences. 2013;22(1):81-91.
17. Molodynski A, Linden M, Juckel G, Yeeles K, Anderson C, Vazquez-Montes M et al. The reliability, validity, and applicability of an English language version of the mini-ICF-APP. Soc Psychiatry Psychiatr Epidemiol. 2013;48:1347-54.

18. Pinna F, Fiorillo A, Tusconi M, Guiso B, Carpiniello B. Assessment of functioning in patients with schizophrenia and schizoaffective disorder with the mini-ICF-APP: a validation study in Italy. Int J Ment Heal Syst. 2015:9:37.

19. Muschalla B. Psychological capacity limitations according to mini-ICF-APP are differently related with sick leave in patients from different professional fields. J Psychosom Res. 2019;124:109741.

20. Muschalla B, Rau H, Willmund GD, Knaevelsrud C. Work disability in soldiers with posttraumatic stress disorder, posttraumatic embitterment disorder, and not-event-related common mental disorders. Psychol Trauma. 2018; 10(1):30-5.

21. Habermeyer B, Kaiser S, Kawohl W, Seifritz E. Assement of incapacity to work and the mini-ICF-APP. Neuropsychiatrie. 2017:31(4):182-6.

22. Canela C, Schleifer R, Dube A, Hengartner MP, Ebner G, Seifritz E, et al. Assessment of functioning when conducting occupational capacity evaluations--what is "evidence-based"? Psychiatr Prax. 2016;43(2):74-81.

23. Nierenberg AA, DeCecco LM. Definitions of antidepressant treatment response, remission, nonresponse, partial response, and other relevant outcomes: a focus on treatment-resistant depression. J Clin Psychiatry. 2001; 62(Suppl 16):5-9.

24. Samara MT, Engel RR, Millier A, Kandenwein J, Toumi M, Leucht S Equipercentile linking of scales measuring functioning and symptoms: examining the GAF, SOFAS, CGI-S, and PANSS. Eur Neuropsychopharmacol. 2014:24(11):1767-72.

25. Leucht S, Kane JM, Etschel E, Kissling W, Hamann J, Engel RR. Linking the PANSS, BPRS, and CGI: clinical implications. Neuropsychopharmacology : official publication of the American College of Neuropsychopharmacology. 2006:31(10):2318-25

26. Bobo WV, Anglero GC, Jenkins G, Hall-Flavin DK, Weinshilboum R, Biernacka JM. Validation of the 17-item Hamilton depression rating scale definition of response for adults with major depressive disorder using equipercentile linking to clinical global impression scale ratings: analysis of Pharmacogenomic research network antidepressant medication Pharmacogenomic study (PGRN-AMPS) data. Hum Psychopharmacol Clin Exp. 2016;31(3):185-92

27. WHO. The ICD-10 classification of mental and behavioural disorders: clinical descriptions and diagnostic guidelines. Geneva: World Health Organization; 1992.

28. Guy W. ECDEU assessment manual for psychopharmacology. Rockville, Maryland: US Department of Health, Education, and Welfare, Public Health Service, Alcohol, Drug Abuse, and Mental Health Administration, National Institute of Mental Health, Psychopharmacology Research Branch, Division of Extramural Research Programs; 1976

29. Spielmans Gl, McFall JP. A comparative meta-analysis of clinical global impressions change in antidepressant trials. J Nerv Ment Dis. 2006;194(11): 845-52.

30. Busner J, Targum SD. The clinical global impressions scale: applying a research tool in clinical practice. Psychiatry. 2007:4(7):28.

31. Rush A Jr, First MB, Blacker DE. Handbook of psychiatric measures Philadelphia: American Psychiatric Publishing, Inc.; 2008.

32. Berk M, Ng F, Dodd S, Callaly T, Campbell S, Bernardo M, et al. The validity of the CGI severity and improvement scales as measures of clinical effectiveness suitable for routine clinical use. J Eval Clin Pract. 2008;14(6): 979-83.

33. WHO. International classification of functioning disability and health (ICF). Geneva: World Health Organization; 2001.

34. Albano AD. equate. Appl Psychol Meas. 2015;40(5):361-2.

35. González J, Wiberg M. Applying test equating methods: using R. Cham, Switzerland: Springer; 2017.

36. Rowland JP, Yeeles K, Anderson C, Catalão R, Morley H, Molodynski A. Is it possible to measure social and occupational functioning in a CMHT? Progress in Neurology and Psychiatry. 2014;18(5):21-5.

37. Duff K. Evidence-based indicators of neuropsychological change in the individual patient: relevant concepts and methods. Arch Clin Neuropsychol. 2012:27(3):248-61.

38. Evans C, Margison F, Barkham M. The contribution of reliable and clinically significant change methods to evidence-based mental health. Evidence Based Mental Health. 1998;1(3):70-2.

39. Schennach-Wolff $R$, Obermeier $M$, Seemüller $F$, Jäger $M$, Schmauss $M$, Laux $\mathrm{G}$, et al. Does clinical judgment of baseline severity and changes in 
psychopathology depend on the patient population? J Clin Psychopharmacol. 2010;30(6):726-31.

40. Leucht $\mathrm{S}$. Measurements of response, remission, and recovery in schizophrenia and examples for their clinical application. J Clin Psychiatry. 2014;75(Suppl 1):8-14.

41. Zimmerman M, Morgan TA, Stanton K. The severity of psychiatric disorders.। World psychiatry : official journal of the World Psychiatric Association (WPA). 2018;17(3):258-75.

42. Gift TE, Strauss JS, Harder DW. The severity of psychiatric disorder. Psychiatry Res. 1980;3(1):31-40.

43. Zimmerman M, Balling C, Chelminski I, Dalrymple K. Understanding the severity of depression: which symptoms of depression are the best indicators of depression severity? Compr Psychiatry. 2018;87:84-8.

44. Faravelli C, Servi P, Arends JA, Strik WK. Number of symptoms, quantification, and qualification of depression. Compr Psychiatry. 1996; 37(5):307-15.

45. Garcia-Velazquez R, Jokela M, Rosenstrom TH. Symptom severity and disability in psychiatric disorders: the U.S. collaborative psychiatric epidemiology survey. J Affect Disord. 2017;222:204-10.

46. Leucht S, Engel RR, Davis JM, Kissling W, Meyer Zur Capellen K, Schmauss M, et al. Equipercentile linking of the brief psychiatric rating scale and the clinical global impression scale in a catchment area. European neuropsychopharmacology : the journal of the European College of Neuropsychopharmacology. 2012;22(7):501-5.

47. Leucht S, Kane JM, Kissling W, Hamann J, Etschel E, Engel R. Clinical implications of brief psychiatric rating scale scores. Br J Psychiatry. 2005; 187:366-71

48. Gross AL, Inouye SK, Rebok GW, Brandt J, Crane PK, Parisi JM, et al. Parallel but not equivalent: challenges and solutions for repeated assessment of cognition over time. J Clin Exp Neuropsychol. 2012;34(7):758-72.

49. Wong A, Black SE, Yiu SYP, Au LWC, Lau AYL, Soo YOY, et al. Converting MMSE to MoCA and MoCA 5-minute protocol in an educationally heterogeneous sample with stroke or transient ischemic attack. International journal of geriatric psychiatry. 2018;33(5):729-34.

50. Wee HL, Yeo KK, Chong KJ, Khoo EYH, Cheung YB. Mean rank, Equipercentile, and regression mapping of World Health Organization quality of life brief (WHOQOL-BREF) to EuroQoL 5 dimensions 5 levels (EQ5D-5L) utilities. Medical decision making : an international journal of the Society for Medical Decision Making. 2018;38(3):319-33.

51. Scheffels JF, Kraling H, Kalbe E, Kessler J. Conversions of cognitive screenings : mini-mental state examination vs. Montreal cognitive assessment vs. DemTect. Nervenarzt. 2018;89(12):1371-7.

52. Saczynski JS, Inouye SK, Guess J, Jones RN, Fong TG, Nemeth E, et al. The Montreal cognitive assessment: creating a crosswalk with the mini-mental state examination. J Am Geriatr Soc. 2015;63(11):2370-4.

53. Leucht S, Fennema H, Engel R, Kaspers-Janssen M, Lepping P, Szegedi A. What does the HAMD mean? J Affect Disord. 2013;148(2-3):243-8.

54. Leucht S, Fennema H, Engel RR, Kaspers-Janssen M, Lepping P, Szegedi A. What does the MADRS mean? Equipercentile linking with the CGI using a company database of mirtazapine studies. J Affect Disord. 2017:210:287-93.

55. Wang PS, Aguilar-Gaxiola S, Alonso J, Angermeyer MC, Borges G, Bromet EJ, et al. Use of mental health services for anxiety, mood, and substance disorders in 17 countries in the WHO world mental health surveys. Lancet. 2007:370(9590):841-50

\section{Publisher's Note}

Springer Nature remains neutral with regard to jurisdictional claims in published maps and institutional affiliations.

Ready to submit your research? Choose BMC and benefit from:

- fast, convenient online submission

- thorough peer review by experienced researchers in your field

- rapid publication on acceptance

- support for research data, including large and complex data types

- gold Open Access which fosters wider collaboration and increased citations

- maximum visibility for your research: over $100 \mathrm{M}$ website views per year

At $\mathrm{BMC}$, research is always in progress.

Learn more biomedcentral.com/submissions 